\title{
Curative effect of combined lamivudine, adefovir dipivoxil, and stem cell transplantation on decompensated hepatitis $B$ cirrhosis
} \author{
H.C. Zhou ${ }^{1}$, Q.M. Wen ${ }^{1}$ and X.M. Wang ${ }^{1}$ \\ ${ }^{1}$ Department of Infectious Disease, Tianyou Hospital, \\ Wuhan University of Science and Technology, Wuhan, China \\ School of Medicine and Health Management, Wuhan, China \\ *These authors contributed equally to this study. \\ Corresponding author: J. Zhou \\ E-mail:1lzjchina@126.com
}

L. Liu ${ }^{1 *}$, Y. Yan ${ }^{2 *}$, J. Zhou ${ }^{1}$, L.W. Huang ${ }^{1}$, C.P. He ${ }^{1}$, K. Ling ${ }^{1}$,

${ }^{2}$ Tongji Medical College of Huazhong University of Science and Technology

Genet. Mol. Res. 13 (4): 9336-9342 (2014)

Received June 18, 2013

Accepted December 6, 2013

Published February 21, 2014

DOI http://dx.doi.org/10.4238/2014.February.21.13

\begin{abstract}
This study assessed the clinical efficacy of lamivudine and adefovir dipivoxil combined with autologous bone marrow stem cell transplantation as treatment for patients with hepatitis B and decompensated liver cirrhosis. In total, 77 patients with hepatitis $\mathrm{B}$ and decompensated liver cirrhosis were randomly divided into two groups. Under general symptomatic and supportive treatment, the patients in group A (37 cases) were treated with lamivudine and adefovir dipivoxil, whereas those in group B (40 cases) were treated with autologous bone marrow stem cell transplantation in combination with lamivudine and adefovir dipivoxil. After 4 weeks of treatment, the liver function indicators and clinical signs and symptoms of the patients in group B improved more significantly than those of patients in group A. Lamivudine and adefovir dipivoxil in combination with autologous
\end{abstract}


bone marrow stem cell transplantation effectively prevented hepatitis B virus infection and bone marrow stem cell damage. This combination treatment facilitates the differentiation of bone marrow stem cells into normal liver cells to restore liver structure and improve liver function, thereby improving the quality of life of patients.

Key words: Lamivudine; Adefovir dipivoxil; Hepatitis B; Autologous bone marrow stem cell; Decompensated liver cirrhosis

\section{INTRODUCTION}

Decompensated hepatitis B cirrhosis is an advanced stage of liver disease. In this stage of liver disease, patients develop many complications and have bad prognosis, and the mortality rate is high. This disease is a serious health threat and is difficult to treat. Persistently high levels and continuous replication of hepatitis B virus (HBV) are extremely important factors for the progression of cirrhosis, and key treatment involves inhibition of viral replication and protection and improvement of liver function.

Lamivudine is a representative anti-HBV drug that has been researched extensively. Clinical studies have shown that it exerts a rapid anti-HBV effect without cytotoxicity and renal toxicity. It can be used to inhibit HBV replication after autologous hematopoietic stem cell transplantation (Silvestri et al., 2000; Henkes et al., 2002; Lin et al., 2005). With an extension of the lamivudine treatment time, variation in HBV DNA polymerase activity zone YMDD is easily induced, which reduces patient sensitivity to lamivudine (Lau et al., 2005). In allogeneic hematopoietic stem cell transplantation, the effect of lamivudine-resistant HBV infection is limited (Ohnishi et al., 2002). Adefovir dipivoxil is a novel anti-HBV nucleoside drug, and its antiviral mechanism is possibly associated with the competitive participation of adenosine monophosphate in the viral DNA chain. As a DNA chain terminator, the drug inhibits DNA polymerase, thus suppressing viral replication (Hadziyannis et al., 2006; Dioguardi et al., 2008). Conversely, it stimulates endogenous interferon A, thereby increasing the activity of natural killer cells and stimulating the immune response. Therefore, it has a stronger anti-HBV effect (Dioguardi et al., 2008). As adefovir dipivoxil affects different target points of DNA polymerase, its antiviral action sites are different from those of lamivudine. Thus, adefovir dipivoxil and lamivudine have no cross-resistance (Takamura et al., 2007). Combination treatment using the two drugs does not cause viral resistance. However, this combination can possibly result in partial drug resistance to adefovir dipivoxil with long-term treatment. Combining adefovir dipivoxil and lamivudine reduces the incidence of adefovir dipivoxil resistance among virus strains. The combination also achieves faster and higher viral responses and a more rapid reduction in the viral replication rates (Liu et al., 2006).

Bone marrow stem cells are an important extrahepatic source of stem cells (Cantz et al., 2004) with multidirectional differentiation ability. In the liver environment, these cells can differentiate into hepatic stem cells and hepatocytes and participate in liver repair and reconstruction and restoration of liver function (Alison et al., 2000; Oh et al., 2000; Theise et al., 2000; Miyazaki et al., 2002; Dahlke et al., 2003; Kania et al., 2004; Levicar et al., 2008). However, after autologous bone marrow stem cells are implanted, they are easily infected and damaged by HBV, which affects their outcomes. Therefore, the present study combined 
lamivudine, adefovir dipivoxil, and autologous bone marrow stem cell transplantation to treat patients with decompensated hepatitis B cirrhosis and analyzed its curative effect.

\section{MATERIAL AND METHODS}

\section{General data}

Seventy-seven patients with decompensated hepatitis B cirrhosis who were hospitalized in the infection department of Tianyou Hospital, Wuhan University of Science and Technology from April 2009 to October 2010 were included in this study. All patients were diagnosed based on the "Viral hepatitis liver cirrhosis diagnosis standard" revised by the Chinese Medical Association in September 2000. The 77 patients were randomly divided into groups A and B. Group A (37 patients) consisted of 33 men and 4 women with a mean age of $50.4 \pm 8.5$ years. Under the Child-Pugh classification, 20 patients had class B cirrhosis, whereas 17 had class C cirrhosis. The duration of disease ranged from 1-15 years. Group B (40 patients) consisted of 37 men and 3 women with a mean age of $51.6 \pm 9.2$ years. Under the Child-Pugh classification, 22 and 18 patients in group $B$ had class $B$ and class $C$ cirrhosis, respectively. The duration of disease ranged from 2 to 18 years. Before treatment, the patients presented different clinical signs and symptoms such as weakness, anorexia, abdominal distension, ascites, hydrothorax, and edema of the lower extremities. All patients were definitively diagnosed with cirrhosis via abdominal ultrasound, computed tomography, or magnetic resonance imaging. This study was conducted in accordance with the declaration of Helsinki, with approval from the Ethics Committee of Tianyou Hospital. Written informed consent was obtained from all participants.

\section{Autologous bone marrow stem cell transplantation}

In the evening before autologous bone marrow stem cell transplantation, the patients were injected subcutaneously with $100 \mu \mathrm{g}$ recombinant human granulocyte colony-stimulating factor. One day before transplantation, the patient's skin at the bilateral groin area was carefully prepared. Four hours before transplantation, the patients discontinued eating and drinking water. At 5-10 $\mathrm{h}$ before bone marrow puncture, the transplantation room was sterilized.

The patients were positioned supine on the operating table. Bone marrow was aspirated via bilateral punctures to the posterior superior iliac spine. After conventional sterilization, the area of the puncture was exposed and locally infiltrated with anesthetic (2\% lidocaine) as deep as the periosteum. Subsequently, an 18-gauge marrow puncture needle was inserted vertically into the marrow cavity and fixed. Two hundred milliliters of bone marrow blood was aspirated from each patient and mixed with $7500 \mathrm{U}$ heparin sodium to prevent coagulation. Then, bone marrow stem cells were isolated and purified using density centrifugation in the stem cell laboratory of our hospital. The resulting cells were counted; the cell concentration was adjusted to $3.2-1.6 \times 10^{10} / \mathrm{mL}$.

One hour before surgery, an iodine hypersensitivity test was conducted. Patients without abnormalities were sent to the intervention room. Under digital subtraction angiography, a puncture cannula was inserted via the femoral artery. When the cannula reached the hepatic inherent artery, angiography was performed to observe the intrahepatic vessels and examine the liver. Then, $10 \mathrm{~mL}$ of the isolated and purified cell suspension was injected slowly via the 
left and right hepatic arteries. At the end of transplantation, the cannula was removed. Pressure dressing was applied to the puncture points. The puncture site was immobilized for $24 \mathrm{~h}$ once the patient was returned to the recovery room.

\section{Observation of curative effect}

The patients were observed for changes in clinical signs and symptoms before transplantation and at 4 weeks after transplantation. Laboratory examinations were conducted before transplantation and at 4 weeks after transplantation, including alanine aminotransferase (ALT), aspartate aminotransferase (AST), total bilirubin (TBIL), plasma albumin (ALB), prothrombin time (PT), and alpha-fetoprotein (AFP) measurements.

\section{Statistical analysis}

The SPSS 13.0 statistical software was used for data analysis, and data are reported as means \pm standard deviation. In addition, a $t$-test was used for comparisons between the two groups. Differences with $\mathrm{P}<0.05$ were considered to be significant.

\section{RESULTS}

\section{Changes in liver function}

After 4 weeks of treatment, the serum AST, ALT, ALB, and TBIL levels of the patients in both groups improved, and the values in group B were significantly higher than those in group A $(\mathrm{P}<0.05$; Table 1$)$.

\begin{tabular}{|c|c|c|c|c|c|}
\hline Group & Cases & ALT (U/L) & $\operatorname{AST}(\mathrm{U} / \mathrm{L})$ & $\operatorname{ALB}(\mathrm{g} / \mathrm{L})$ & TBIL $(\mu \mathrm{M})$ \\
\hline \multicolumn{6}{|l|}{ A } \\
\hline Before treatment & 37 & $72.3 \pm 21.5$ & $90.2 \pm 26.8$ & $32.2 \pm 3.9$ & $59.2 \pm 17.6$ \\
\hline After treatment & 37 & $52.7 \pm 13.4 *$ & $65.3 \pm 18.5^{*}$ & $33.0 \pm 3.0$ & $41.3 \pm 12.7 *$ \\
\hline \multicolumn{6}{|l|}{ B } \\
\hline Before treatment & 40 & $75.1 \pm 19.8$ & $87.7 \pm 24.6$ & $31.9 \pm 3.7$ & $61.1 \pm 16.5$ \\
\hline After treatment & 40 & $35.2 \pm 12.5^{* \#}$ & $48.3 \pm 13.7 * \#$ & $35.8 \pm 3.1$ *\# & $30.8 \pm 11.9^{\text {*\# }}$ \\
\hline
\end{tabular}

\section{Changes in PT, cholinesterase (CHE), and AFP}

After 4 weeks of treatment, PT, CHE, and AFP values of the patients in both groups improved, and the values in group $\mathrm{B}$ were significantly higher than those in group $\mathrm{A}(\mathrm{P}<0.05$; Table 2).

\section{Clinical symptoms and signs}

After 4 weeks of treatment, the clinical signs and symptoms of the patients in both groups were clearly improved, and significantly more patients in group B had improved clinical symptoms than in group $\mathrm{A}(\mathrm{P}<0.05$; Table 3$)$. 
Table 2. Change in prothrombin time (PT), cholinesterase (CHE), and alpha-fetoprotein (AFP) values in the two groups before and after treatment.

\begin{tabular}{|c|c|c|c|c|}
\hline Group & Cases & PT (s) & CHE (U/L) & $\mathrm{AFP}(\mathrm{ng} / \mathrm{mL})$ \\
\hline \multicolumn{5}{|l|}{ A } \\
\hline Before treatment & 37 & $24.5 \pm 3.0$ & $75.5 \pm 21.3$ & $12.6 \pm 5.4$ \\
\hline After treatment & 37 & $23.4 \pm 2.1$ & $87.7 \pm 18.7$ & $13.7 \pm 6.7$ \\
\hline \multicolumn{5}{|l|}{ B } \\
\hline Before treatment & 40 & $24.7 \pm 2.8$ & $78.2 \pm 23.5$ & $13.1 \pm 6.0$ \\
\hline After treatment & 40 & $19.0 \pm 2.2^{* \#}$ & $103.5 \pm 20.4^{* *}$ & $24.7 \pm 9.7 * \#$ \\
\hline
\end{tabular}

$* \mathrm{P}<0.05$ for comparison of the two groups before and after treatment. ${ }^{*} \mathrm{P}<0.05$ for comparison of the two groups after treatment.

\begin{tabular}{|c|c|c|c|c|c|c|}
\hline Group & Cases & Pleural fluid & Ascitic fluid & Abdominal distension & Low limb edema & Fatigue, anorexia \\
\hline \multicolumn{7}{|l|}{$\overline{\mathrm{A}}$} \\
\hline Before treatment & 37 & 10 & 24 & 32 & 30 & 34 \\
\hline After treatment & 37 & 7 & 18 & 24 & 22 & 27 \\
\hline \multicolumn{7}{|l|}{ B } \\
\hline Before treatment & 40 & 11 & 26 & 33 & 31 & 35 \\
\hline After treatment & 40 & $3^{* *}$ & $9^{* *}$ & $15^{* *}$ & $12 * *$ & $14^{* * *}$ \\
\hline
\end{tabular}

*P $<0.05$ for comparison of the two groups before and after treatment. ${ }^{\#} \mathrm{P}<0.05$ for comparison of the two groups after treatment.

\section{Adverse reactions}

During treatment, the two groups of patients had no severe adverse reactions and complications. After autologous bone marrow stem cell transplantation therapy, one patient in group B developed a low-grade fever, two developed nausea, and one developed an oozing wound. The symptoms were clearly improved after symptomatic supportive treatment.

\section{DISCUSSION}

Decompensated hepatitis B cirrhosis is an advanced stage of liver disease. This stage is associated with many complications, a poor prognosis, and high mortality rates. It is a serious health threat and is difficult to treat. Persistently high HBV levels and continuous HBV replication are extremely important factors in the progression of cirrhosis, and key treatment involves inhibiting viral replication as well as protecting and improving liver function (Silvestri et al., 2000).

Lamivudine is a representative anti-HBV drug that has been researched extensively. Clinical studies have shown that this drug has a rapid anti-HBV effect, and does not induce cytotoxicity and renal toxicity. Extending lamivudine treatment easily induces variations in HBV DNA polymerase activity zone YMDD. The mutated gene sequence of DNA polymerase coded by viral DNA occurred near the sequence of YMDD. So it is called YMDD variation, which reduces sensitivity to lamivudine (Lau et al., 2005). Adefovir dipivoxil is a novel antiHBV nucleoside drug, and its antiviral mechanism is possibly associated with the competitive participation of adenosine monophosphate in the viral DNA chain. As a DNA chain terminator, it inhibits DNA polymerase, thereby suppressing viral replication (Ohnishi et al., 2002; Hadziyannis et al., 2006). On the contrary, endogenous interferon A increases the activity of 
natural killer cells and stimulates the immune response. Therefore, it has a stronger anti-HBV effect (Dioguardi et al., 2008). As adefovir dipivoxil affects different target points of DNA polymerase, its antiviral action sites are different from those of lamivudine. Thus, adefovir dipivoxil and lamivudine have no cross-resistance (Takamura et al., 2007). Regarding combination application of the two drugs, there is no obvious virus resistance phenomenon. The effect of this combination is possibly related to partial resistance to adefovir dipivoxil that develops during long-term treatment. The combination of adefovir dipivoxil and lamivudine reduces the adefovir dipivoxil resistance of viral strains, induces faster and higher viral responses, and more rapidly reduces the viral replication rate.

Autologous bone marrow stem cell transplantation was performed in addition to lamivudine and adefovir dipivoxil treatment for decompensated hepatitis B cirrhosis. Recent studies revealed that autologous bone marrow stem cell transplantation significantly improves cirrhosis (Wollert et al., 2004; am Esch 2nd et al., 2005). This treatment has the following advantages: 1) the cells required for surgery are easier to obtain and not restricted or influenced by the lack of donors. Using autologous bone marrow cells prevents postoperative immunologic rejection (Deans and Moseley, 2000; Tae et al., 2006; Heino and Hentunen, 2008). 2) The curative effect of this procedure is more obvious (Henkes et al., 2002). 3) The cost of this procedure is relatively low. In China, the mean cost of autologous bone marrow stem cell transplantation therapy ranges between $¥ 3000$ and $¥ 5000$, which is comparatively lesser than that of liver transplantation, whereas its curative effect, especially the long-term effect, is not significantly different from that of liver transplantation. 4) The technological risk of this procedure is small. Bone marrow extraction is simple and minimally invasive.

The laboratory indicators and clinical signs and symptoms in the two groups improved after 4 weeks of treatment, and the serum AST, ALT, ALB, TBIL, PT, CHE, and AFP values improved more significantly in group $\mathrm{B}$ than in group $\mathrm{A}(\mathrm{P}<0.05)$. In addition, significantly more patients in group B exhibited improvements in clinical signs and symptoms than in group A $(\mathrm{P}<0.05)$. This result suggests that the addition of autologous bone marrow stem cell transplantation improves liver function, coagulation, AFP levels, and the clinical signs and symptoms of patients. No severe adverse reactions and complications were observed among the patients during and after the surgery. Moreover, the liver volume and amount of liver blood flow of some patients clearly increased.

Therefore, combining lamivudine, adefovir dipivoxil, and autologous bone marrow stem cell transplantation minimizes the risk of HBV infection in bone marrow stem cells in the treatment of decompensated hepatitis B cirrhosis. The combination treatment also helps bone marrow stem cells to differentiate into normal hepatic cells in the liver and participate in the repair and reconstruction of the liver to improve liver function, thereby improving the quality of life of the patients.

\section{REFERENCES}

Alison MR, Poulsom R, Jeffery R, Dhillon AP, et al. (2000). Hepatocytes from non-hepatic adult stem cells. Nature 406: 257. am Esch JS 2nd, Knoefel WT, Klein M, Ghodsizad A, et al. (2005). Portal application of autologous CD133+ bone marrow cells to the liver: a novel concept to support hepatic regeneration. Stem. Cells 23: 463-470.

Cantz T, Sharma AD, Jochheim-Richter A, Arseniev L, et al. (2004). Reevaluation of bone marrow-derived cells as a source for hepatocyte regeneration. Cell Transplant. 13: 659-666.

Dahlke MH, Popp FC, Bahlmann FH, Aselmann H, et al. (2003). Liver regeneration in a retrorsine/ $\mathrm{CCl}_{4}$-induced acute liver failure model: do bone marrow-derived cells contribute? J. Hepatol. 39: 365-373. 
Deans RJ and Moseley AB (2000). Mesenchymal stem cells: biology and potential clinical uses. Exp. Hematol. 28: 875884.

Dioguardi N, Grizzi F, Fiamengo B and Russo C (2008). Metrically measuring liver biopsy: a chronic hepatitis B and C computer-aided morphologic description. World J. Gastroenterol. 14: 7335-7344.

Hadziyannis SJ, Tassopoulos NC, Heathcote EJ, Chang TT, et al. (2006). Long-term therapy with adefovir dipivoxil for HBeAg-negative chronic hepatitis B for up to 5 years. Gastroenterology 131: 1743-1751.

Heino TJ and Hentunen TA (2008). Differentiation of osteoblasts and osteocytes from mesenchymal stem cells. Curr. Stem. Cell Res. Ther. 3: 131-145.

Henkes M, Martin S, Einsele H and Aulitzky WE (2002). Successful antiviral treatment for fulminant reactivated hepatitis B after autologous stem cell transplantation and prophylaxis during subsequent allogeneic stem cell transplantation. Ann. Hematol. 81: 343-346.

Kania G, Blyszczuk P, Jochheim A, Ott M, et al. (2004). Generation of glycogen- and albumin-producing hepatocyte-like cells from embryonic stem cells. Biol. Chem. 385: 943-953.

Lau GK, Piratvisuth T, Luo KX, Marcellin P, et al. (2005). Peginterferon Alfa-2a, lamivudine, and the combination for HBeAg-positive chronic hepatitis B. N. Engl. J. Med. 352: 2682-2695.

Levicar N, Pai M, Habib NA, Tait P, et al. (2008). Long-term clinical results of autologous infusion of mobilized adult bone marrow derived CD34+ cells in patients with chronic liver disease. Cell Prolif. 41 Suppl 1: 115-125.

Lin PC, Poh SB, Lee MY, Hsiao LT, et al. (2005). Fatal fulminant hepatitis B after withdrawal of prophylactic lamivudine in hematopoietic stem cell transplantation patients. Int. J. Hematol. 81: 349-351.

Liu CJ, Kao JH, Chen PJ, Chen TC, et al. (2006). Overlap lamivudine treatment in patients with chronic hepatitis B receiving adefovir for lamivudine-resistant viral mutants. J. Viral Hepat. 13: 387-395.

Miyazaki M, Akiyama I, Sakaguchi M, Nakashima E, et al. (2002). Improved conditions to induce hepatocytes from rat bone marrow cells in culture. Biochem. Biophys. Res. Commun. 298: 24-30.

Oh SH, Miyazaki M, Kouchi H, Inoue Y, et al. (2000). Hepatocyte growth factor induces differentiation of adult rat bone marrow cells into a hepatocyte lineage in vitro. Biochem. Biophys. Res. Commun. 279: 500-504.

Ohnishi M, Kanda Y, Takeuchi T, Won KS, et al. (2002). Limited efficacy of lamivudine against hepatitis B virus infection in allogeneic hematopoietic stem cell transplant recipients. Transplantation 73: 812-815.

Silvestri F, Ermacora A, Sperotto A, Patriarca F, et al. (2000). Lamivudine allows completion of chemotherapy in lymphoma patients with hepatitis B reactivation. Br. J. Haematol. 108: 394-396.

Tae SK, Lee SH, Park JS and Im GI (2006). Mesenchymal stem cells for tissue engineering and regenerative medicine. Biomed. Mater. 1: 63-71.

Takamura M, Ichida T, Ohkoshi S, Tsubata S, et al. (2007). Decompensated lamivudine-resistant hepatitis B virus-related cirrhosis treated successfully with adefovir dipivoxil allowing surgery for hepatocellular carcinoma. Intern. Med. 46: 367-371.

Theise ND, Badve S, Saxena R, Henegariu O, et al. (2000). Derivation of hepatocytes from bone marrow cells in mice after radiation-induced myeloablation. Hepatology 31: 235-240.

Wollert KC, Meyer GP, Lotz J, Ringes-Lichtenberg S, et al. (2004). Intracoronary autologous bone-marrow cell transfer after myocardial infarction: the BOOST randomised controlled clinical trial. Lancet 364: 141-148. 